\title{
Transgenic zebrafish forms technicolour 'skinbow'
}

Fluorescent proteins used to track individual skin cells in real time.

\section{Adam Levy}

21 March 2016

\section{Skinbow}

3D view of the outer layer of cells in a regenerating zebrafish fin.

Researchers have created a transgenic zebrafish with skin that fluoresces in thousands of colours - enabling them to track the behaviour of hundreds of individual cells in real time and to see what happens to skin when it is wounded.

"It's a spectacular-looking fish," says Kenneth Poss, who studies tissue regeneration at Duke University in Durham, North Carolina, and who led the research, published in Developmental Cell ${ }^{1}$.

The idea is based on the 'brainbow' - a technique published in $2007^{2}$ in which neurons were engineered to express mixtures of fluorescent proteins, producing a random combination of different colours in different cells.

\section{Chen et al./Developmental Cell 2016}

An adult 'skinbow' zebrafish, seen under a microscope in normal lighting conditions.

In a zebrafish engineered to express the proteins in its skin cells, the same concept is called the 'skinbow'. The fluorescent cells are limited to the fish's outer layer of skin, and cover the entire surface of its body - even the cornea.

\section{Reading the rainbow}

Based on the number of combinations of red, green and blue proteins they can express, individual cells could be expected to shine in any one of around 5,000 different colour combinations. In fact, only around 70 colours can be distinguished clearly through a microscope. Still, that is enough for most cells to be 'barcoded' distinctly from their neighbours.

By tracking hundreds of individual skin cells over time, the researchers were able to study in real time how the skin recovered from 
injury, such as a scrape or a fin amputation.

Chen et al./Developmental Cell 2016

A dissected scale from a 'skinbow' zebrafish. Skin cells shining in multiple fluorescent colours cover the top layer of the scale.

The scientists found that when a fin is amputated, for example, the cells employ three distinct processes to keep regenerating tissue covered with skin. Skin cells from neighbouring regions migrate in to cover the new tissue; new skin cells are created and some cells also grow substantially.

"The interesting thing about this kind of study," says Poss, "is you don't know what to expect, but you have a great way to visualize it."

Nature | doi:10.1038/nature.2016.19615

\section{References}

1. Chen, C.-H. et al. Dev. Cell 36, 668-680 (2016).

2. Livet, J. et al. Nature 450, 56-61 (2007). 